\title{
Numerical applications for experimental IRT in defective multilayered building systems
}

\author{
by C. Serra ${ }^{*, * *}$, A. Tadeu ${ }^{*, * *}$, and N. Simões ${ }^{*, * *}$ \\ * ITeCons - Institute for Research and Technological Development for Construction, Energy, Environment and \\ Sustainability, Rua Pedro Hispano s/ n., 3030-289 Coimbra, Portugal, cserra@itecons.uc.pt \\ **ADAI - LAETA, Department of Civil Engineering, University of Coimbra, Pólo II, Rua Luís Reis Santos, 3030- \\ 788 Coimbra, Portugal
}

\begin{abstract}
Active Infrared Thermography (IRT) tests are conducted on test specimens made from materials commonly used in building elements which contain thin defects located at various depths. The results are analysed using a phase contrast approach. Then, numerical simulations will be carried out using a boundary elements method (BEM) model formulated in the frequency domain to model 3D heat diffusion in a multilayered system containing a thin defect. The purpose of this work is to compared and discuss the numerically and experimentally obtained phase contrast results and therefore provide numerical applications for experimental IRT in defective multilayered building systems.
\end{abstract}

\section{Introduction}

Infrared Thermography (IRT) is currently being used in many areas to evaluate materials and to detect and characterize defects located beneath the surface [1]. The quantitative characterization of such defects requires the employment of specific testing and processing techniques, which involve the application of numerical simulation models. Most known active IRT techniques ([2],[3]) were developed for the non-destructive evaluation of thin highly conductive materials. Therefore, such techniques are generally not suited for IRT applications in buildings, since construction materials and products generally have lower conductivity and require deeper probing. Among the available techniques, pulse-phase ([4],[5]) based ones have been suggested to be the most adequate for buildings since phase images are able to provide better defect geometry resolution. Furthermore, longer heating enables probing at greater depths in materials such as concrete, mortar, etc. [6].

The interest in performing predictive maintenance in ageing buildings and structures to cut down the cost of repairs is growing, as is the need to improve energy efficiency. Hence, there can be said that there is an increasing need for further research into IRT applications in buildings. Since many constructive systems and building elements follow a layered configuration, the interest in studying heat transfer in multilayered buildings is also becoming more significant. The work presented herein aims to contribute to the interpretation of experimental IRT results obtained in building inspections by providing experimental and numerical results. An experimental campaign is performed using test specimens that replicate defective building elements and a 3D heat diffusion simulation model (previously developed by the authors [7]) for layered media containing thin defects is then used. The numerically and experimentally obtained phase contrast results will later be compared and discussed.

\section{Methodology}

A number of active IRT experiments are performed on test specimens that contain thin defects located at different depths. In these tests, the specimens are subjected to thermal stimulation using a long pulse and thermal images are recorded using an IRT camera. In order to take advantage of the benefits presented by phase images, both numerical and experimental results are presented in terms of phase contrast images and phase contrast curves. Thermal wave phase is calculated by applying a Fourier transform to the temperature results recorded in each pixel of the thermal image. Phase contrast is extracted by selecting a pixel located in an area which is considered not to be influenced by the defect (sound area) to act as a reference. By extracting phase contrast for a specific receiver (corresponding to a pixel in the image captured by an IRT camera) phase contrast curves, which are a graphical representation that can be used to characterize a given defect, are generated.

The model which will be used to compute 3D heat transfer by conduction in defective layered media is based on a boundary element method (BEM) formulated in the frequency domain and incorporates analytical solutions for multilayered media derived by Tadeu et al. ([8],[9]) which are expressed as Bessel integrals, following the procedure proposed by Sommerfeld ([10],[11]). This model only requires the discretization of the defect's surface since the 3D Green's functions for multilayered media avoid the discretization of the interfaces between layers. A detailed formulation of this model has been previously presented by the authors [7]. Since the model is written in the frequency domain, numerical thermal wave phase results are computed directly. Concurrently, the thermal wave phase response in a sound medium (without defects) is computed using a purely analytical model. Phase contrast is given by the difference between these two responses. 
Looking to illustrate the usefulness of the model in active IRT building applications, both numerical and experimental results for defects located at different depths will be compared and discussed.

\section{Experimental results}

Active IRT tests were performed using a FLIR A615 camera connected to a control unit (Automation Technology IRX-Box) and to a computer with IR NDT 1.74 software. The IRT camera has the following technical specifications: $640 \times 480$ pixels resolution; $7.5-14 \mu \mathrm{m}$ spectral range; field of view (FOV) of $25^{\circ} \times 19^{\circ} ; 0.68 \mathrm{mrad}$ spatial resolution; thermal sensitivity/noise equivalent temperature difference (NETD) $<0.05^{\circ} \mathrm{C} @ 30{ }^{\circ} \mathrm{C}$ and accuracy of $\pm 2{ }^{\circ} \mathrm{C}$ or $2 \%$. The tests were performed in reflection mode with the camera $0.90 \mathrm{~m}$ away from the surface being inspected and using two $2500 \mathrm{~W}$ halogen lamps. The resulting FOV of the recorded images is $0.389 \times 0.300 \mathrm{~m}^{2}$.

The thermal stimulation was performed following a rectangular heating function. In each test, thermal images were recorded for a defined length of time (total test duration of either 4096s and $8192 \mathrm{~s}$ ), which includes an initial offset period (100 s), a heating period ( $200 \mathrm{~s}$ ) and a cooling down period (remainder of the recording time). A total of either 128 or 256 thermal images were recorded in each test, corresponding to an acquisition frequency of $0.03125 \mathrm{~Hz}(128 \mathrm{frames}$ recorded for $4096 \mathrm{~s}$ and 256 frames recorded for $8192 \mathrm{~s}$ ).

Three test specimens were constructed to simulate defective building elements with thin inclusions located at three different depths: $d=0.01 \mathrm{~m}, d=0.02 \mathrm{~m}$ and $d=0.04 \mathrm{~m}$. Each test specimen was assembled by pouring a cementbased mortar mix into a box constructed with a marine plywood, and by carefully placing a $0.05 \times 0.05 \mathrm{~m}^{2}$ defect made from $0.0022 \mathrm{~m}$ thick polyethylene foam at the specific desired depth. An insulation layer of $0.04 \mathrm{~m}$ (extruded polystyrene foam) was placed along the lateral boundaries of the specimen in order to minimize heat flow exchange. For each pixel in the FOV, phase results were calculated by applying a Fourier transform to the thermal wave results recorded in the time domain. Phase contrast was then determined by computing the difference between the phase recorded in each pixel and the phase recorded at a receiver located in a sound area (one that is not influenced by the defect).

The following image illustrates the experimental phase contrast results obtained in the campaign.

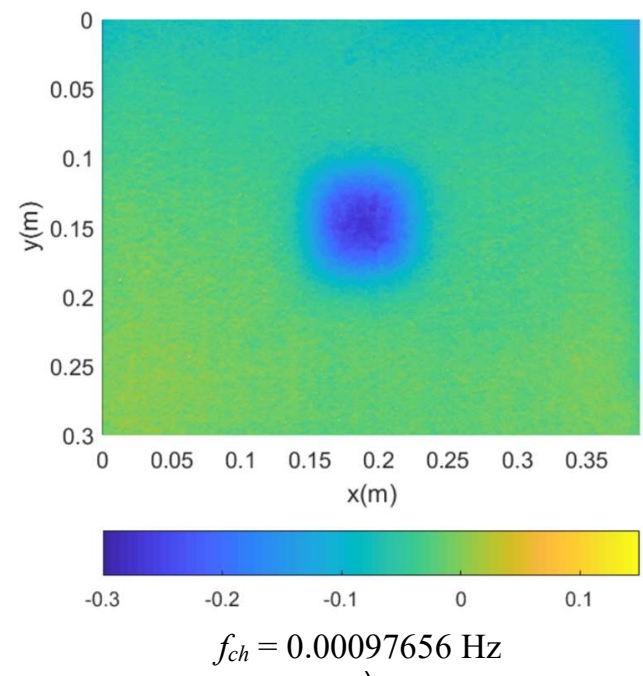

a)

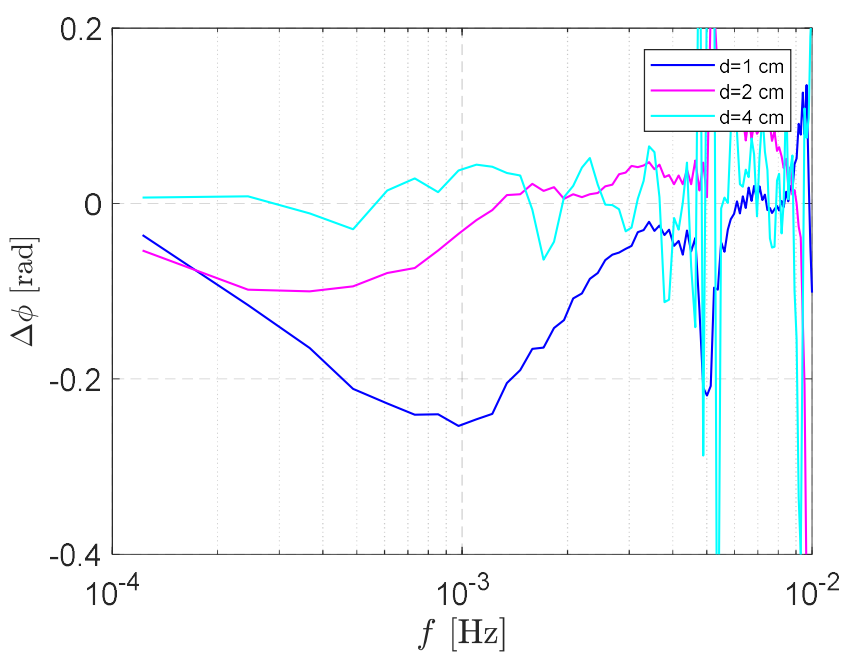

b)

Fig. 1. Phase contrast results: a) image for the $(d=1 \mathrm{~cm})$ specimen at maximum visibility; b) curves that characterize defects located at different depths.

On the left is a phase contrast image recorded at the frequency in which the $(d=0.01 \mathrm{~cm})$ defect is most visible. This is also known as the characteristic frequency $f_{c h}$. On the right are the phase contrast curves obtained for the three test specimens with different defect depths $(d=0.01 \mathrm{~m}, d=0.02 \mathrm{~m}$ and $d=0.04 \mathrm{~m})$, for a receiver located at the centre of the image (corresponding to the centre of the defect). Each of the shallower defect curves $(d=0.01 \mathrm{~m}, d=0.02 \mathrm{~m})$ show a clear absolute maximum phase contrast $(|\Delta \phi \max |)$. The deeper defect curve does not, indicating loss of visibility in terms of phase contrast image.

\section{Final remarks}

The purpose of this work is to provide numerical applications for active IRT tests in defective building elements. Numerical simulations will be carried out using a boundary elements method (BEM) model formulated in the frequency domain to model $3 \mathrm{D}$ heat diffusion in a multilayered system containing a thin defect. Both numerical and experimental results for defects located at different depths will be compared and discussed. 


\section{Acknowledgements}

This work was framed within the POCI-01-0247-FEDER-017889 (INOVWALL) Project funded by Portugal 2020 through the COMPETE 2020.

\section{REFERENCES}

[1] Maldague X. Theory and Practice of Infrared Technology for Non-destructive Testing. John Wiley \& Sons, 2001.

[2] Maldague X. Introduction to NDT by Active Infrared Thermography. Materials Evaluation 2002;6(9),1060-1073.

[3] Ibarra-Castanedo C, González D, Klein M, Pilla M, Vallerand S, Maldague X. Infrared image processing and data analysis. Infrared Physics \& Technology 2004;46(1-2):75-83.

[4] Maldague X, Marinetti S. Pulse phase infrared thermography, Journal of Applied Physics 1996;79(5):26942698.

[5] Ibarra-Castanedo C, Maldague X. Pulsed phase thermography reviewed. Quantitative InfraRed Thermography Journal 2004;1(1):47-70.

[6] Arndt RW. Square pulse thermography in frequency domain as adaptation of pulsed phase thermography for qualitative and quantitative applications in cultural heritage and civil engineering. Infrared Physics \& Technology, 2010;53(4):246-253.

[7] Serra C, Tadeu A, Simões N. Boundary element method simulation of 3D heat diffusion in defective layered media for IRT building applications. Engineering Analysis with Boundary Elements 2017;81:44-52.

[8] Tadeu A, António J, Simões N. 2.5D Green's functions in the frequency domain for heat conduction problems in unbounded, half-space, slab and layered media. Computer Modeling in Engineering and Sciences - CMES, 2004;6(1):43-58

[9] Tadeu A, Simões N. Three-dimensional fundamental solutions for transient heat transfer by conduction in an unbounded medium, half-space, slab and layered media. Engineering Analysis with Boundary Elements 2006;30(5):338-349.

[10] Sommerfeld A. Mechanics of deformable bodies, New York: Academic Press, Inc., 1950.

[11] Ewing WM, Jardetzky WS, Press F. Elastic Waves in Layered Media, McGraw-Hill Book Company, 1957. 